\title{
DEVELOPMENT OF AHP BASED MODELS AND DSS FOR STRATEGIC PLANNING OF LOCAL SUSTAINABLE DEVELOPMENT
}

\author{
Zaneta Servini ${ }^{*}, \mathrm{PhD}$ candidate, \\ University St. Kliment Ohridski, \\ Bitola, Macedonia, \\ E-mail: hzaneta@yahoo.com \\ Igor Nedelkovski, $\mathrm{PhD}$ \\ University St. Kliment Ohridski, \\ Bitola, Macedonia, \\ E-mail: igor.nedelkovski@gmail.com \\ Jani Servini, Bsc IT engineer, \\ PSTS Gjorgji Naumov, \\ Bitola, Macedonia, \\ E-mail: serjani@yahoo.com
}

\begin{abstract}
A model of a decision support system (DSS) for strategic planning of municipal development with appropriate original software is developed in this paper. A multi-criteria decision-making model, specifically, the analytical hierarchy model (AHP) methodology for model development is applied. The problem of insufficient effectiveness and efficiency of decision making in implementing strategic planning (SP) of local sustainable development (SD) through local agenda 21 (LA21) process is analyzed. It was decided that the most appropriate form for its solution is application of group DSS (GDSS), conducted with the proper software support. The problem of choosing between the alternatives has been solved using two AHP models for priority problems and actions determining. The most important criteria and sub-criteria have been considered. The created software offers a possibility of conducting sensitivity analysis. It will provide information about sensitiveness of alternatives priority according to changes in input weights of the criteria.
\end{abstract}

Keywords: Social issues application, GDSS, AHP, LA21 strategic planning, decision making

\section{Introduction}

The process of strategic planning (SP) of local sustainable development (SD), implemented through a Local Agenda 21 (LA21) process, which is accepted and supported by the UN, involves identifying the priority problems of the citizens, and proposing actions to overcome them. It is evidently a need for active involvement of representatives of all stakeholders at the local level: local government (LG), civic organizations (COs) and business sector (BS), whose interests often differ with each other. If we add to this more stressed requirement for transparency, it becomes clear that there is a great need to accelerate the decision-making processes with many representatives of the stakeholders that most appropriately can be realized by developing an appropriate decision support system (DSS). The main purpose of the DSS is to improve the quality and efficiency of decision-making. Incidental usage of DSS in the public sector in recent years has been identified as a problem that requires special treatment and solution for many

\footnotetext{
* Corresponding author
} 
reasons. One of them is the fact that local development has a significant impact on the qua lity of citizens' life and the other is the fact that many interested parties have conflicting interests: local representatives from different political parties are involved in local decision-making. More specifically, this paper proposes the creation of AHP (analytical hierarchy process) models and appropriate purpose-oriented software products that will allow the application of DSS in the two essential phases of LA21 process (given in Figure 1) which include prioritizing and ranking of local problems and actions.

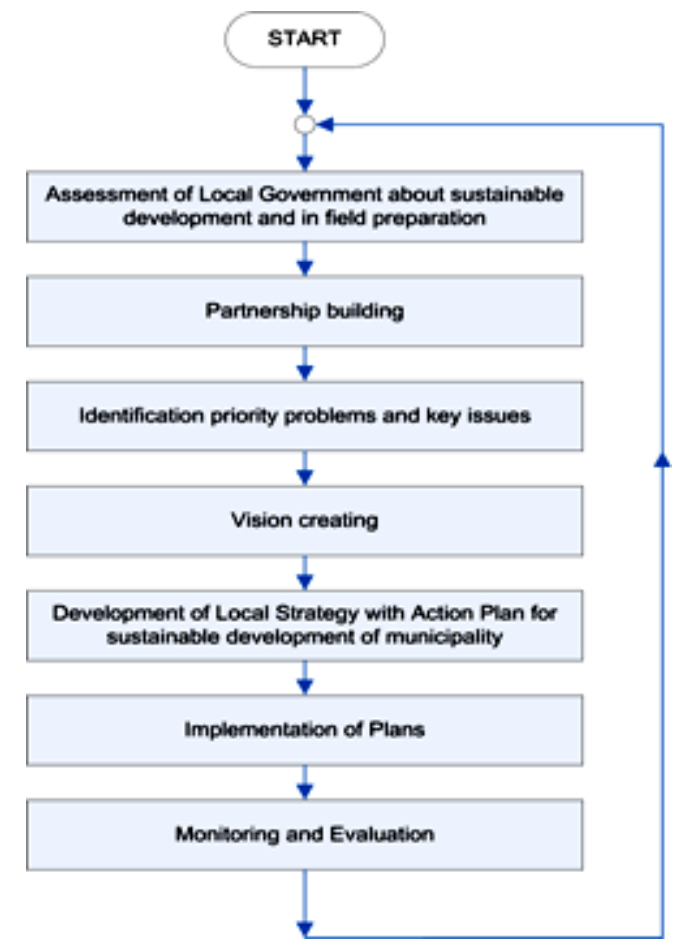

Figure 1. Phases of LA21 process.
The problems are a result of the uncontrolled use of resources and the unmet needs of citizens in the munic ipality. To get to the list of problems, (which will be prioritized), it is necessary to generate the Lists (Inventories) of local resources and unmet needs. By examining and determining the quality and quantity of resources in the community and the impact of the manner of their use, the local government will have a starting point for the preparation and implementation of plans, programs and projects for their effective management. In this respect, every resource should be analyzed in terms of three aspects: pressure, state and response. Namely, each of these aspects concerns the state of the resource, the cause of that situation and the actions, which have been taken on its improvement. To analyze each resource, its regular measurement and data collection is required. In terms of sustainability, human needs can be classified into four categories of needs in the area of: economic, environmental, social sphere and the sphere of management. Since the citizens' opinion is very important for the implementation of this step, research of public opinion includes conducting a questionnaires survey in the munic ipality.

Questions should address the unmet needs and they should be correlated with a list of resources. Problematic areas and key municipal issues will appear with analysis of the survey results of the needs and resources state. After determining the list of problems, follows DSS application for their prioritization. Bearing in mind the comprehensiveness of LA21, stakeholders should recommend a number of actions to achieve general goals and specific objectives. On the other hand, usually limited financial and human resources dictate a need for the implementation of several priority actions. Deciding which action is more suitable for realization than another is not a simple process, especially when we know that the actions differ in their scope, activities, duration and necessary financial, material and human resources and representatives of different parties should make a decision. Considering this, actions can be ranked according to the mutually agreed criteria.

\section{Development of AHP models}

Two AHP models are defined within this paper: one on the ranking of local problems (priority issues), and the second, ranking of priority actions. These models, as a part of DSS, will effectively increase implementation of the strategic planning process of local sustainable development, although there are other decision-making phases of the LA21 process, where the system can be applied, such as the stage of determining the overall goals and specific objectives. 
To generate the AHP model, by following the general steps, it is necessary to define a goal, criteria, subcriteria and alternatives. In that aim, it is necessary to define a team of experts. In our case, the expert team consisted of five evaluators, experienced in project management, strategic and action planning and implementation of DSS. Experts joined to design hierarchy of MADM problem, and to determine relative weights of the criteria by comparison of pairs, applying the AHP model for assessment. First, they developed hierarchy for the MADM problem and got AHP models for ranking of priority issues and actions. Then they set weights of major criteria and sub-criteria. A team of experts used specially designed AHP based software for decision-making support based on comparisons of pairs of relative weights of each criterion and sub-criteria to calculate the weights of the criteria and sub criteria. We had in mind that the group can generate more ideas and generally, it has a greater knowledge than individuals have and objectivity enhances by group decision making. In our case, five experts accepted the result of group AHP. However, if the effect of polarization appears, which treats the appearance of risk and caution in making decisions, we recommend applying the 3-rd phase of AHP (von Solms, S., 2003).

\subsection{AHP model (hierarchy) of criteria for problem prioritization}

The aim of the first AHP model shown in Figure 2 is to rank priority problems in the specific munic ipality for which the local government will develop a strategic plan according to the LA21 process.

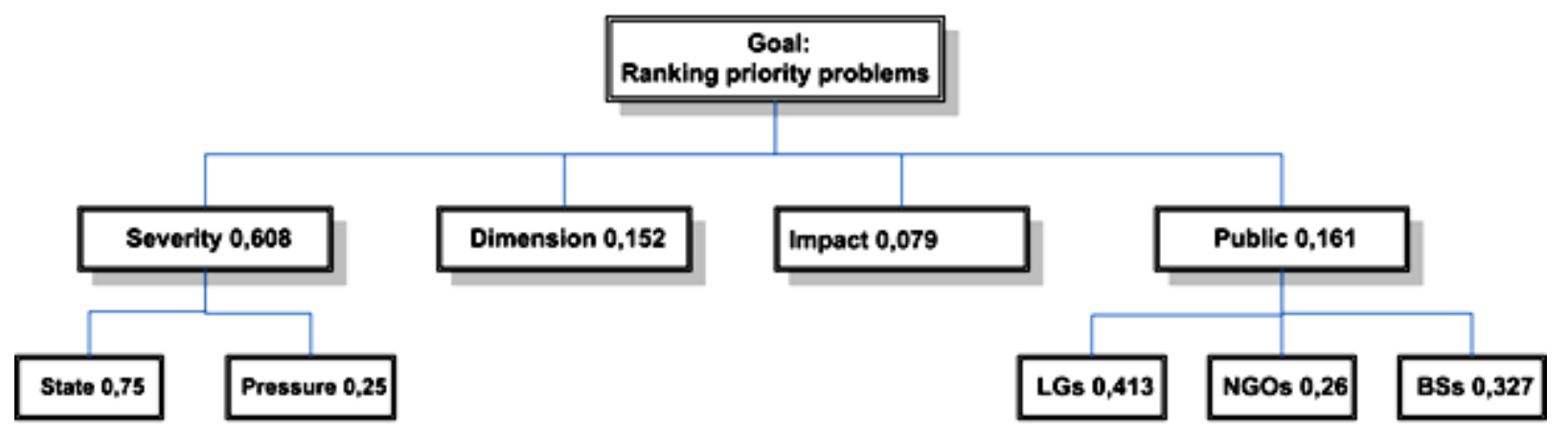

Figure 2. General hierarchical model for determining the priority problems.

Taking in consideration the LA21 methodology, the hierarchy of the criteria is:

a) Severity (seriousness) of the particular problem, defined by two sub-criteria: $a_{1}$ ) state - specific data and facts that define the current state of this particular problem, and $\mathrm{a}_{2}$ ) pressure - data that describe the assumed dynamics (change) of the state of the problem, i.e. its future enlargement.

b) Dimension (size, scope) of the specific problem defined by the number of affected residents who are concerned with the specific problem, and the extent (bounds) of geographic region.

c) Negative impact of the problem as a criterion means how the particular problem affects the creation of other problems in the municipality.

d) Public opinion about the given problem comprises different categories of representatives. It is the opinion of representatives from various sectors for the present problem and is defined by three sub-criteria: $\mathrm{d}_{1}$ ) local government, $\mathrm{d}_{2}$ ) civic organizations (COs), and $\mathrm{d}_{3}$ ) business sector.

In the hierarchical model given above the public opinion is a quantitative criterion obtained through surveys, as averages of individual scores of all surveyed participants, taking into account their specific values in the comparisons. For an efficient application, a simplified model can be used, without using public opinion as a criterion and without the two sub-criteria of the criteria severity._In this case, stakeholders' representatives will conduct a survey with all the groups mentioned above and will receive a preliminary problem priority list. It will be the result of the analyses of 40-50 problems and will locate 5-10 priority problems that have to be ranked with applying the AHP methodology. In that way, with a group DSS, the final priority is determined. 


\subsection{AHP model (hierarchy) of criteria for priority actions}

The purpose of the second AHP model hierarchy, given in Figure 3, is determining the priority actions in the actual municipality, for which a strategic plan for local sustainable development is created. From the list of actions, it is necessary to choose the ones with the highest priority. For this purpose, according to LA21 and SWOT analyses, an expert team defined the following criteria:

so Existence of resources, with four sub-criteria, defined as follows, 80 financial resources - whether financial resources are provided to implement the action,

8 human resources - whether human resources are provided for implementing the action,

\&o time resources - the time needed for implementation of the action,

\&o equipment and premises as resources - whether material and technical resources are provided for implementing the action;

sos Sustainability of the action, with four sub-criteria, defined as follows:

so local economy - whether the action helps to solve the economic problem,

so social welfare - whether the action helps to solve the problem in the domain of social welfare (health, education, welfare, sports, recreation, culture),

\&o local governance - whether the action helps to solve the problem of local governance,

80 environmental protection - whether the action helps to solve the problem in the domain of environmental protection;

so Appropriateness of the action, defined by three sub-criteria as follows:

so effectiveness - whether the action solves the problem, achieving a desired result (achieving the defined specific and general goals),

80 efficiency - whether satisfactory results are achieved or objectives are reached without wasting too many resources (money, time ...),

so flexibility - whether the action can be modif ied after a certain period of time to adjust to any economic, demographic, environmental conditions and legal frameworks).

For the actions' priority ranking, when a simplified model is used, the sub-criteria of criterion resources will be excluded.

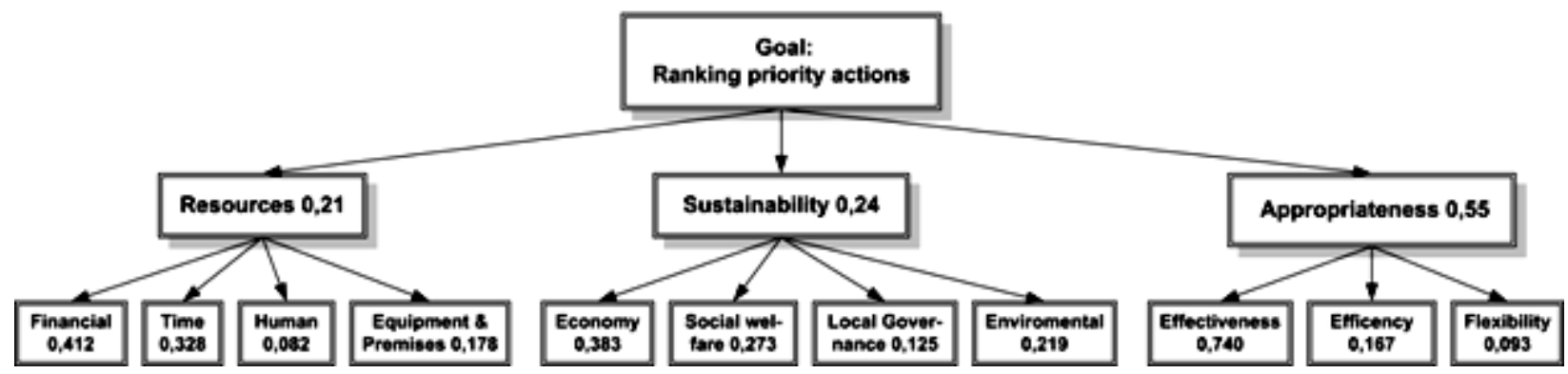

Figure 3. AHP model (hierarchy) of criteria for priority actions.

At the bottom of the hierarchy, stakeholders define alternatives, whose priorities should be set. After that, they compare pairs of offered alternatives in relation to the criteria in the matrix. The matrix of relative relations of weights of the criteria and sub-criteria of optimality and the matrixes of preference of the alternatives in relation to each sub-criteria of optimality are input data to determine the best alternative, taking into account all criteria of optimality and their weights simultaneously.

Quantitative criteria are included in comparisons with their values, and the qualitative criteria weights are compared based on Saaty's scale of intensity. The consistency of matrices is checked (allowed inconsistency is up to 0.10). First, individual assessments are received, and after that, they are combined into a group model, which synthesize the priorities. In the last step of the proposed AHP models, the alternatives that have relatively higher total scores of priority will be identified as the most important and 
they should be analyzed. Decision makers can perform a sensitivity analys is, which shows how the priority of alternatives changes, depending on the change of weights of any of the criteria.

\section{Char acteristics (features) of DSS software}

From the analysis of researches in the world, authors derive an important conclusion that in AHP methodology application a commercial software system, e.g Expert choice is usually used. It was an additional motivation for authors to initiate research on the creation of AHP models, which are then used in developing new DSS software. The generated system, except in the specific research field, has a much wider application. The system is developed and implemented in Microsoft.NET platform.

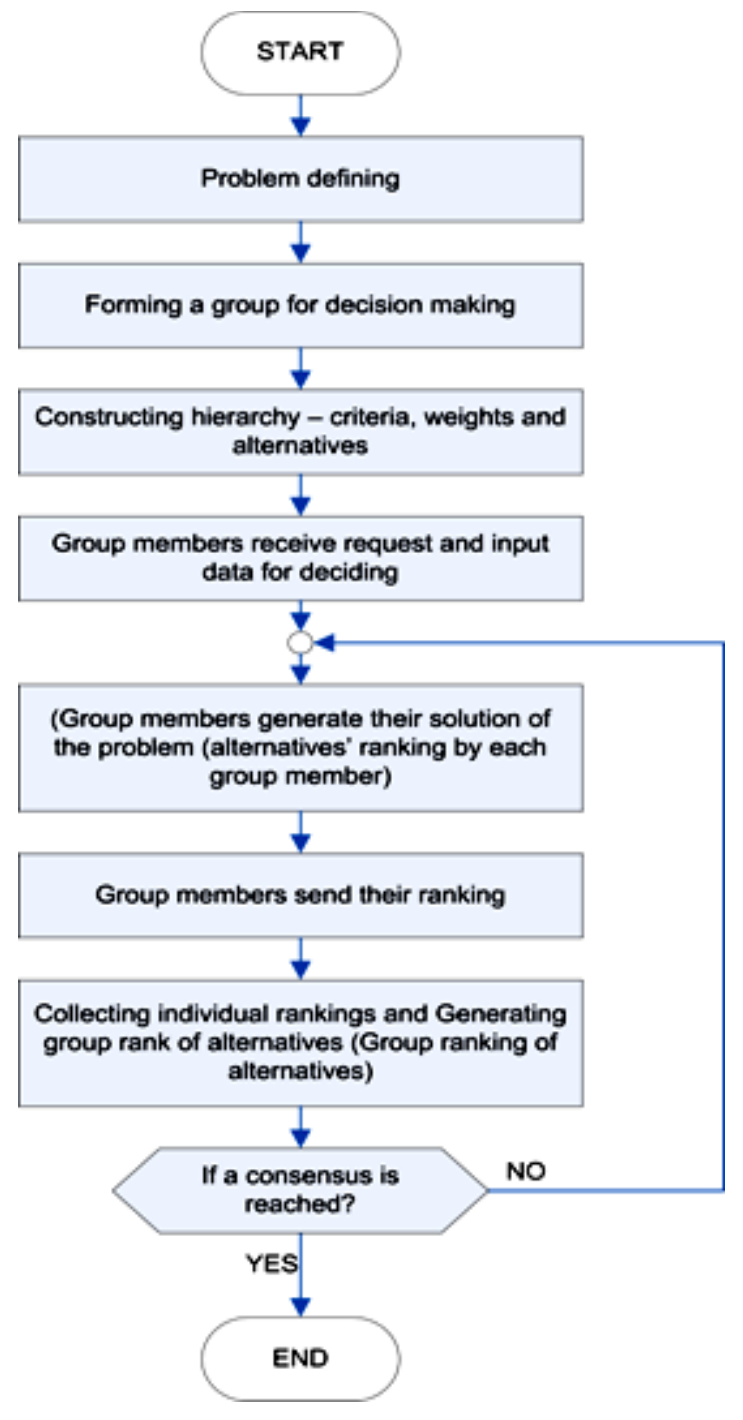

Figure 4. Working process flowchart.

Global aspect of the working process of DSS is presented in Figure 4. DSS can be accessed via the interface component as an administrator or evaluator. The administrator has special privileges and he sets the input parameters: the group members, their levels (weights), then the decision making model, criteria and their weights, alternatives and data that describe them. The administrator submits a request to each of the evaluators to decide and to rank the alternatives according to given criteria. Each of them logs into the system as a member of the group and, taking into account the input data, received by the administrator, performs the ranking of alternatives by priority, based on the AHP method. The evaluator fills in the appropriate matrix scores and gets a solution in the form of priority ranking, which he sends to the administrator. After receiving individual priority ranking of each member of the evaluation team, the administrator accesses to DSS and performs group ranking. Finally, the resulting rankings are presented to all decision makers, who discuss it through normal debate or through Delphilike method and after reaching consensus about the group AHP priority, that solution proposed by DSS will be accepted. If consensus is not reached, then is recommended to implement another AHP (feedback in Figure 4), elaborated in Von Solms, S. (2001) as phase 3, which will be accepted as a final solution. In our case, there was only one individual and one group AHP, as well as a consensus discussion, where DSS results were accepted.

The actual software was tested and applied in real conditions for preparing the strategy for regional development between four municipalities in four Balkan countries, as a part of major project, approved by the European Commission (Nedelkovski I., Servini Z. and Servini J, 2011). The received results were equal to results of Expert Choice 2000 in third decimal. It was a challenge for us to develop new and original, simple and user-friendly software, with exact results as EC, and from a language aspect with different possibilities than EC. 


\section{Conclusion}

Strategic planning of local government is a broad process, which involves decision making by multiple stakeholders. In this paper the AHP method was selected as most suitable for creating a model and software development - group system to support decision making in strategic planning, because:

so it includes a number of criteria with different weights,

sos it is suitable for ranking a number of alternatives depending on several criteria,

sos it provides an opportunity to measure the consistency and to conduct sensitivity analysis. Additionally, by applying AHP methodology, effectiveness and efficiency are increased. Effectiveness, because they will get a real priority, which will reflect the opinion of each evaluator, who has the knowledge and power, i.e. expert and decision-maker in municipality, and efficiency, because all resources are reduced to minimum, especially time needed for decision making. This model and software, based on AHP method, can be used in any kind of prioritizing in different fields of social life, i.e. for:

is preparation of strategic plans based on sustainable development on a regional and national level,

it development of strategic plans for the business sector,

is decision-making in local government in public procurements and

is sustainable management of natural resources in a region.

For future researches, we recommend improving of the model and the software (modeling and development of WEB AHP based group DSS).

\section{REFERENCE:}

De Moraes, E. A. \& Bernardes, R.C. (2001). Project Portfolio Management using AHP. Centro Universitário da FEI São Paulo, SP Brazil

Nedelkovski I., Servini Z. and Servini J. (2011). Application of AHP based DSS for strategic planning of local sustainable development. International Symposium on the Analytic Hierarchy Process 2011, Sorento, Italy

Saaty, T. L. (1999). How to Make and justify a Decision: The Analytic Decision Process. University of Pittsburgh

Servini J. (2004). Guide for Local Agenda 21. Ministry for environmental and physical planning, Republic of Macedonia

Sprague, R.H. and Watson, H.J. (1993). Decision Support Systems: Putting Theory into Practice, 3rd Edition, Prentice-Hall, London

Von Solms, S. (2003). Group Polarization, Social Influence and the Analytic Hierarchy Process. Proceedings 7th International Symposium on the Analytic Hierarchy Process; Bali, Indonesia; August; $475-484$

Von Solms, S. \& Peniwati, K. (2001). An Empirical Comparison of Two Choice Aggregation Methods in the AHP. Proceedings 6th International Symposium on the Analytic Hierarchy Process; Bern, Switzerland; August; 541-549 